\title{
Gastric Cancer in Young Patients Under The Age of 30 Years
}

\author{
Prochorov ${ }^{*}{ }^{*}$, Labunec $\mathrm{I}^{1}$, Kazakevich $\mathrm{V}^{2}$, Shepetjko $\mathrm{M}^{1}$ and Papok $\mathrm{V}^{1}$ \\ ${ }^{1}$ Department of Oncology, Belarusian State Medical University, Minsk, Belarus \\ ${ }^{2}$ Department of Oncosurgery \#4, Minsk City Clinical Oncological Hospital, Minsk, Belarus
}

${ }^{*}$ Corresponding author: Prochorov A, Head of Department of Oncology, Belarusian State Medical University, Dzerzhinski ave., 83, Minsk, Belarus, 220116, E-mail: aprokharau@gmail.com

Citation: Prochorov A, Labunec I, Kazakevich V, Shepetjko M, Papok V (2015) Gastric Cancer in Young Patients Under The Age of 30 Years. J Cancer Sci Clin Oncol 2(1): 102. doi: 10.15744/2394-6520.1.202

Received Date: January 20, 2015 Accepted Date: March 04, 2015 Published Date: March 09, 2015

\begin{abstract}
A retrospective analysis was conducted on 8466 gastric cancer patients from different age groups treated in Minsk City Clinical Oncological Hospital from 1998 to 2013. Of these, $72(0.85 \%)$ patients were under the age of $30,72.2 \%$ of which were diagnosed with clinical stage IV cancer. Radical surgery was performed on 17 patients (24.0\%), palliative on $11(15.0 \%)$ and symptomatic on 22 (31.0\%). Out of these 72 patients, $75 \%$ had a three-year survival rate, while $62 \%$ had five-year survival rate. Overall survival rate after palliative surgery did not exceed two years. Without specific antitumor treatment all patients under the age of 30 with advanced stomach cancer, died within a year, while adjuvant chemotherapy increased the life span by not more than a year.

Keywords: Gastric cancer; Surgical treatment; Results of treatment; Young patients under the age of 30 years
\end{abstract}

\section{Introduction}

Despite some progress in early detection and treatment, gastric cancer remains one of the most complex healthcare and socioeconomic problems. Belarus is among the countries with the highest incidence rate of cancer with this localization. In 2013, according to the Belarusian National Cancer Registry, 2888 new cases of gastric cancer were registered, with peak incidence observed in the 75-79 age group; men were affected more frequently than women at a ratio of 2.4:1. In 2013 in Belarus the five-year survival rate was 23.5\%; and for patients with clinical stage I and II - it was 50.3\% [1].

Over the last decade the incidence rate of gastric cancer in young patients has a trend towards a gradual increase [1-5]. As the first clinical signs of early gastric cancer are nonspecific, and its symptoms are very similar to that of gastritis and gastric ulcer, the diagnosis is often made late. Previous studies indicate that tumor develops within 4-6 years; however, the recorded medical history usually does not exceed one year, indicating an extensive asymptomatic period. This is why many questions regarding the molecular-biological conditions, early diagnosis and treatment of stomach cancer at a young age still remain relevant and not well-researched [6-9].

The purpose of this study was to examine the characteristics of clinical development as well as anatomo-morphologic characteristics of the tumor, and to evaluate the results of multimodal treatment of gastric cancer patients under the age of 30 .

\section{Patients and methods}

From 1998 to 2013, 8466 patients with malignant stomach tumors were registered in Minsk. Out of that sample, 77 (0.9\%) patients were under the age of 30. Gastric cancer was diagnosed in 72 cases $(0.85 \%)$ that accounted for $93.5 \%$ of total amount of malignant incidents; carcinoid was diagnosed in 3 cases (3.9\%) and gastrointestinal stromal tumor was confirmed in 2 cases (2.6\%).

Evaluation of long-term results of treatment (including postoperative mortality) and comparison of survival rates, depending on different variables in groups, were conducted by Kaplan-Meier method and the log-rank test. The long-term results were calculated based on the analysis of the survival rates following treatment of 68 patients. The fate of 4 patients with gastric cancer in stage IV without special treatment is unknown (left the Republic of Belarus).

\section{Results}

In the observed group of 72 young gastric cancer patients under the age of 30, male to female ratio was 1:1.5. According to the Belarusian National Cancer Registry, overall male to female ratio for all age groups was 2.4:1. So, for young adults under the age of 30, female population is more predisposed to gastric cancer than male. In the 15-20 age group, gastric cancer was diagnosed in 3 cases (4.2\%), in 21-25 age group - in 20 cases (27.8\%) and in 26-29 age group- in 49 (68.0\%) cases. In all cases the diagnosis was confirmed by histological tests. Glandular and poorly differentiated forms of gastric cancer were prevailing (Table 1). 


\begin{tabular}{|l|l|l|l|}
\hline Tumor histological type & Abs. (\%) & Tumor Grade & Abs. (\%) \\
\hline Adenocarcinoma & $37(51.4 \%)$ & Well- differentiated G1 & $5(6.9 \%)$ \\
\hline Signet ring cell carcinoma & $18(25.0 \%)$ & Moderately differentiated G2 & $6(8.3 \%)$ \\
\hline Undifferentiated carcinoma & $17(23.6 \%)$ & Poorly differentiated G3 & $44(61.1 \%)$ \\
\hline & & Anaplastic G4 & $17(23.6 \%)$ \\
\hline Total & $72(100 \%)$ & & $72(100 \%)$ \\
\hline
\end{tabular}

Table 1: Patients' distribution by tumor histological structure and grade

Patient distribution by the extent of cancer spread (TNM Classification 2007) is presented in Table 2.

\begin{tabular}{|l|l|l|l|}
\hline T index: abs. (\%) & N index: abs. (\%) & M index: abs. (\%) & Stage: abs. (\%) \\
\hline T1 6 (8.3\%) & N0 20 (27.8\%) & M0 22 (30.6\%) & I 9 (12.5\%) \\
\hline T2 11 (15.3\%) & N1 23 (31.9\%) & M1 50 (69.4\%) & II $4(5.6 \%)$ \\
\hline T3 34 (47.2\%) & N2 24 (33.4\%) & & III 7 (9.7\%) \\
\hline T4 21 (29.2\%) & N3 5 (6.9\%) & & IV 52 (72.2\%) \\
\hline Total: $72(100 \%)$ & Total: 72 (100\%) & Total: 72 (100\%) & Total: 72 (100\%) \\
\hline
\end{tabular}

Table 2: Patients' distribution by the extent of cancer spread

In overwhelming majority (72.2\%) young patients were diagnosed with an advance stage of gastric cancer. Total or sub-total stomach lesion was confirmed in $59.7 \%$ cases, distal part of the stomach was affected in 17 cases (23.6\%), proximal part - in 12 (16.6\%), and spreading of the tumor to the esophagus was confirmed in 7 cases (9.7\%). Adjacent organs were affected in $76.4 \%$ of cases; lymphatic and hematogenous metastases were confirmed in $72.2 \%$ and $69.4 \%$ cases, respectively. Peritoneal carcinomatosis with malignant ascites condition was reported in $41.7 \%$ cases. Analysis of the family medical history revealed that $23.5 \%$ of patients in observation had a close family member with cervical cancer, breast cancer, colon cancer or prostate cancer. However, as no causal relationship between these family incidents and developing of gastric cancer in the observed group of young patients was identified, all the cases under study were considered non-hereditary and sporadic.

Out of 72 cases only in 28 (38.9\%) curative extirpation or resection of the stomach was possible to perform. Extirpation of the stomach was performed on 12 patients (incl. 1 case with the resection of the lower third of the esophagus by Garlock-Osawa), proximal gastrectomy - on 4 patients (incl. 2 cases with resection of the lower third of the esophagus by Garlock-Osawa) and distal subtotal gastrectomy on 12 patients. In 5 cases resection or extirpation was combined with resection or removal of adjacent organs (liver, pancreas and spleen).

Out of 28 patients that were treated with extirpation or resection of the stomach, 17 (60.7\%) underwent radical surgery with the standard D2 lymphadenectomy, which included the removal of at least 25 regional lymph nodes. Eleven patients in clinical stage IV with local carcinomatosis and clinical complications (perforation, bleeding, tumor stenosis), underwent palliative subtotal resection or gastrectomy.

For 22 patients the surgery procedure was limited to diagnostic laparoscopy in 10 cases, to exploratory laparotomy in 7 cases and to symptomatic surgery with bypass gastroenteroanastomosis and gastro-or jejunostomy in 5 cases. In 22 cases of asymptomatic gastric cancer with no clinical complications and morphologically confirmed invasion of adjacent organs (multiple metastases in the liver, carcinomatous ascites), curative surgery was not possible to perform. Following explorative and symptomatic surgery, 2 patients died postoperatively from the progression of the disease. Mortality after radical and palliative resections and gastrectomies was not observed. Analysis of the morphological tests results indicates that gastric cancer in young patients is characterized by the development of mostly aggressive low differentiated forms of the disease (84.7\%) and, consequently, exhibits more aggressive biological behavior with the locoregional cancer progression and distant metastasis (in $72.2 \%$ cases), which reduces the possibility of radical curative surgery and leads to worse prognosis results.

In our study early diagnosis of gastric cancer in young patients has become possible due to regular check - up screenings for gastritis and peptic duodenal ulcer.

27 patients were treated with postoperative adjuvant chemotherapy ( 2 to 8 cycles of ELF): in 5 cases - after radical surgery of locally metastatic tumor, in all cases - after palliative resections and extirpation of the stomach ( 5 cases with adjuvant radiation therapy with $40 \mathrm{~Gy}$ ), in 11 cases of 22 - after the diagnostic laparotomy or symptomatic surgery. 9 patients with stage IV out of 22 were treated with chemotherapy without the surgery. In other cases the treatment was limited to symptomatic conservative therapy.

Long-term results of gastric cancer treatment, arranged by the scope of the surgery, are presented in Figure 1.

Analysis of these results revealed that after the radical surgery 11 patients (74.7\%) survived a 3-year span, 6 (62.5\%) patients - a 5 -year span, including 4 cases with no signs of recurrence for more than 7 years, and 1 case - for more than 12 years. Median survival time after radical operations amounted to 97.1 months, after palliative - to 8.4 months, after symptomatic- to 4.2 months. The median survival time without the surgery was 3.1 months. 


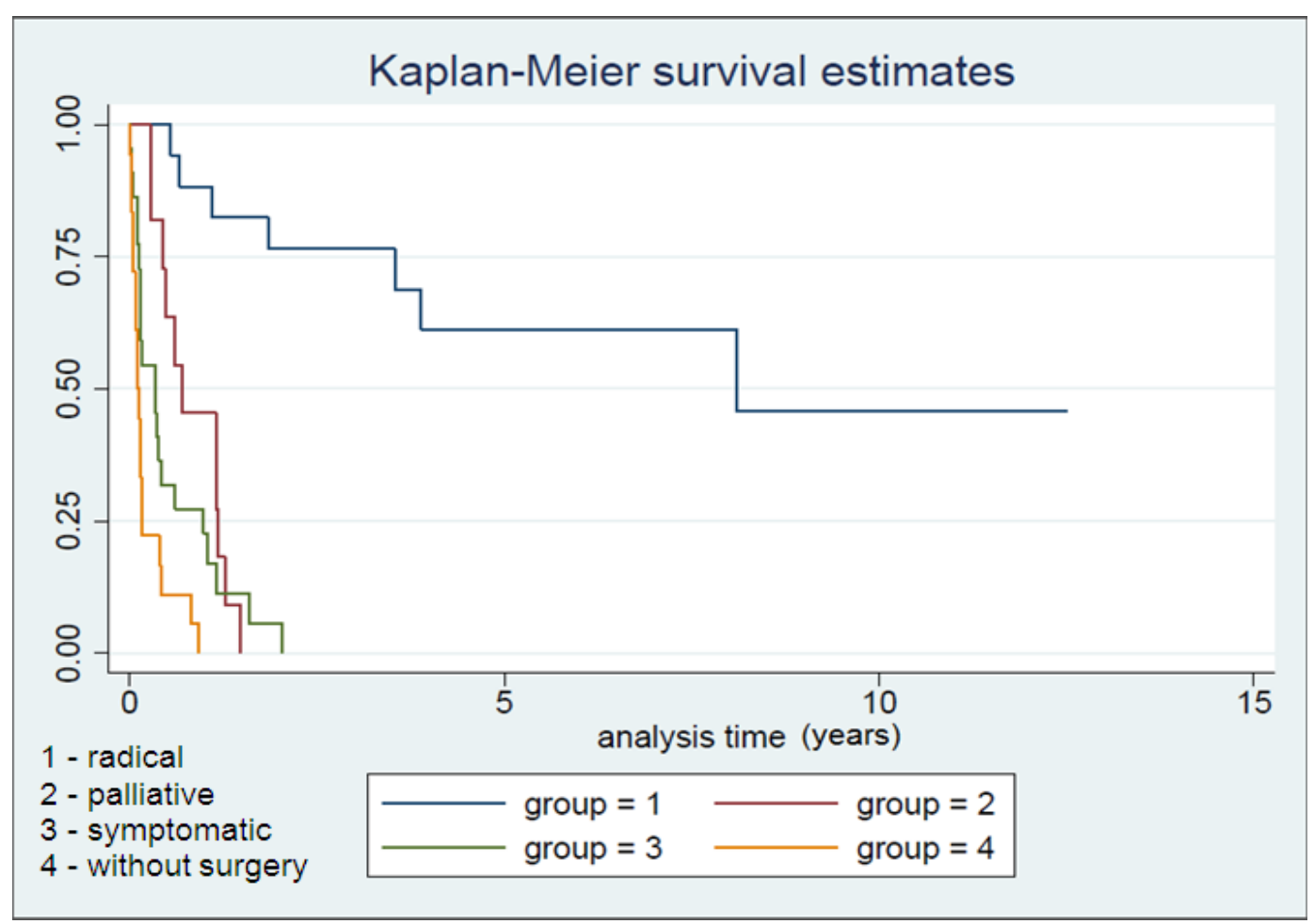

Figure 1: Survival rates depending on the scope of the surgery

In 11 cases of palliative resections and extirpations of the stomach, all patients died within the first two years of observation, regardless of being treated or not with adjuvant chemotherapy. In fact, 6 of them did not survive one year. In comparison, 87.5\% of patients (35 of 40), diagnosed with an advanced stage and treated with antineoplastic and symptomatic care, including those who underwent exploratory and symptomatic surgery, died within 1 year; the rest - within 2 years. Patients who did not undergo chemotherapy died within one year after being diagnosed. For those who received antineoplastic treatment, one-year mortality was $75.0 \%$, while the rest of the group survived for up to 2 years. However, the difference in survival rates is not statistically significant due to a small sample size.

\section{Discussion}

Belarus is among the countries with the highest rate of gastric cancer (30.5/100000) and the highest mortality rate (20.4/100000). The overall 5-year survival rate in 2013 was 23.5\%; and for the patients diagnosed with stage I and II it was 50.3\%. Unfortunately, early diagnostics of gastric cancer in stages I and II remains unsatisfactory, accounting only for $40.9 \%$ of cases. According to our most recent records, gastric cancer incidence rate for patients under the age of 30 is 2.4/100000 without any evidence of significant downward trend (in 2009 2.9/100000) [1]. The data indicates dramatically higher incidence rates of cancer in females than in males (3.3/100000 and 1.6/100000, respectively). According to various studies, such a trend is observed in many regions of the world $[9,10]$, although the research of López-Basave H.N. et al. indicates that male to female ratio is 1:1 [7]. Our observations are consistent with the data of other authors, demonstrating that the young patients tend to have more aggressive undifferentiated forms of gastric cancer, most frequently presented by a signet ring cell carcinoma, followed by an early and rapid metastasis [712]. In our study we did not conduct a special genetic analysis of observed patients, therefore, all the cases were considered to be sporadic. However, it is worth mentioning that $23.5 \%$ of patients under study had family history of cancer with other localizations. The study by Buffart T.E. et al. indicates that young, middle-aged and elderly patients have different genome profiles, which are strongly correlated with age [6]. These findings could be used as a base for further genetic research of young group of patients [7].

Our study illustrated that $72.2 \%$ of young patients first admitted to the hospital were diagnosed with gastric cancer in stage IV, which eliminated the possibility of radical curative treatment. Only 17 of 72 patients were treated with radical resection or gastrectomy with lymph node dissection D2. Previous research has documented early metastasis in young patients [7,10,11,13]. Out of 72 patients only 13 were diagnosed with gastric cancer in stage I and II due to regular check-up and monitoring for other stomach chronic diseases. The reason of the late diagnosis of gastric cancer in the young adults is the lack of programs for early detection of resectable gastric cancer, which is consistent with the opinion of other authors [7-9].

Some studies indicate that the results of radical treatment of young patients, diagnosed with gastric cancer in early stages, are similar to those of older patients [8,9]. In contrast to this opinion Smith B.R. and Stabile B.E. found that average survival times for young and for older patients were 11.6 months and 33.4 months, respectively [13]. Yet, our analysis did not show that age can be used as a predictor to survival rates; instead we believe that the major predictive factors for a prognosis are the histological type of the tumour, lymph node lesion and adequate curative surgery [11]. 
A very important issue is the development of the effective protocols for adjuvant therapy for patients with a high risk of cancer progression after palliative surgeries. Our observations revealed that chemotherapy treatment is not statistically significant in increasing survival rates for patients under the age of 30, and we believe that a feasible way of increasing the survival rate in young patients is further development of neoadjuvant medication therapy that requires additional randomized studies.

It should be mentioned, that an achievable short-term solution to the increase of 5-year survival rate in young patients could be establishing a program for early gastric cancer diagnosis based on diagnostic endoscopy with biopsy and new methods of comprehensive treatment $[7,12]$.

\section{Conclusion}

The incidence rate of gastric cancer in young patients under age of 30 is relatively low comparatively to that of older patients and does not exceed $1 \%$. The incidence rate is dramatically higher for young females than for young males, with the female to male ratio being 1.5:1.

Most frequently, at the moment young patients first diagnosed with gastric cancer, it is already in its advanced stage. Moreover, predominance of undifferentiated forms of tumor and peritoneal metastasis limit the possibility of radical curative surgery. The major reason for the disease progressing to such an advanced level is the delay in diagnosis. After radical surgery 3-year gastric cancer survival rate in young patients is $74.5 \%$, 5-year - $62.5 \%$; overall survival rate after palliative surgery does not exceed 2 years. Further research is required to improve the results of gastric cancer treatment in young patients. Specifically, early diagnostic screening through endoscopy might be one of the possible solutions to explore. As adjuvant chemotherapy is not significant in increasing of the life span after the palliative surgery, further study of the neoadjuvant chemotherapy for gastric cancer treatment would be beneficial.

\section{References}

1. Okeanov AE, Moiseyev PI, Levin LF (2014) Statistics of cancer diseases in the Republic of Belarus (2004-2013) Edited by Sukonko OG N. N. Alexandrov National Cancer Centre of Belarus 69-75.

2. Shah MA, Kelsen DP (2010) Gastric cancer: a primer on the epidemiology and biology of the disease and an overview of the medical management of advanced disease. J Natl Compr Canc Netw 8: 437-47.

3. Merrill RM, Sloan A, Anderson AE, Ryker K (2011) Unstaged cancer in the United States: a population-based study. BMC Cancer 11: 402.

4. Babak O, Protas Yu (2005) Chronic atrophic gastritis - point reference start carcinogenesis. Modern Gastroentero 5: 9-14.

5. Al-Refaie WB, Hu CY, Pisters PW, Chang GJ (2011) Gastric adenocarcinoma in young patients: a population-based appraisal. Ann Surg Oncol 18: 2800-7.

6. Buffart TE, Carvalho B, Hopmans E, Brehm V, Kranenbarg EK, et al. (2007) Gastric cancers in young and elderly patients show different genomic profiles. J Pathol 211: 45-51.

7. Lopez-Basave HN, Morales-Vasquez F, Ruiz-Molina JM, Namendys-Silva SA, Vela-Sarmiento I, et al. (2013) Gastric cancer in young people under 30 years of age: worse prognosis, or delay in diagnosis? Cancer Manag Res 5: 31-6.

8. Park YK, Kim JC, Koh YS, Joo JK, Ryu SY, et al. (2006) Early gastric carcinoma in young patients. Int Surg 91: 316-9.

9. Isobe T, Hashimoto K, Kizaki J, Miyagi M, Aoyagi K, et al. (2013) Characteristics and prognosis of gastric cancer in young patients. Oncol Rep 30: 43-9.

10. Hiroaki S, Takaya S, Fukumoto Y, Osaki T, Tatebe S, et al. (2012) Clinicopathologic Characteristics and Prognosis of Gastric Cancer in Young Patients. Yonago Acta Med 55: 57-61.

11. Kim DY, Joo JK, Ryu SY, Park YK, Kim YJ, et al. (2005) Clinicopathologic characteristics of gastric carcinoma in elderly patients: a comparison with young patients. World J Gastroenterol 11: 22-6.

12. Isik M, Caner S, Metin Seker M, Civelek B, Odabas H, et al. (2011) Gastric adenocarcinoma under the age of 40; more metastatic, less differentiated. J BUON 16: $253-6$.

13. Smith BR, Stabile BE (2009) Extreme aggressiveness and lethality of gastric adenocarcinoma in the very young. Arch Surg 144: 506-10.

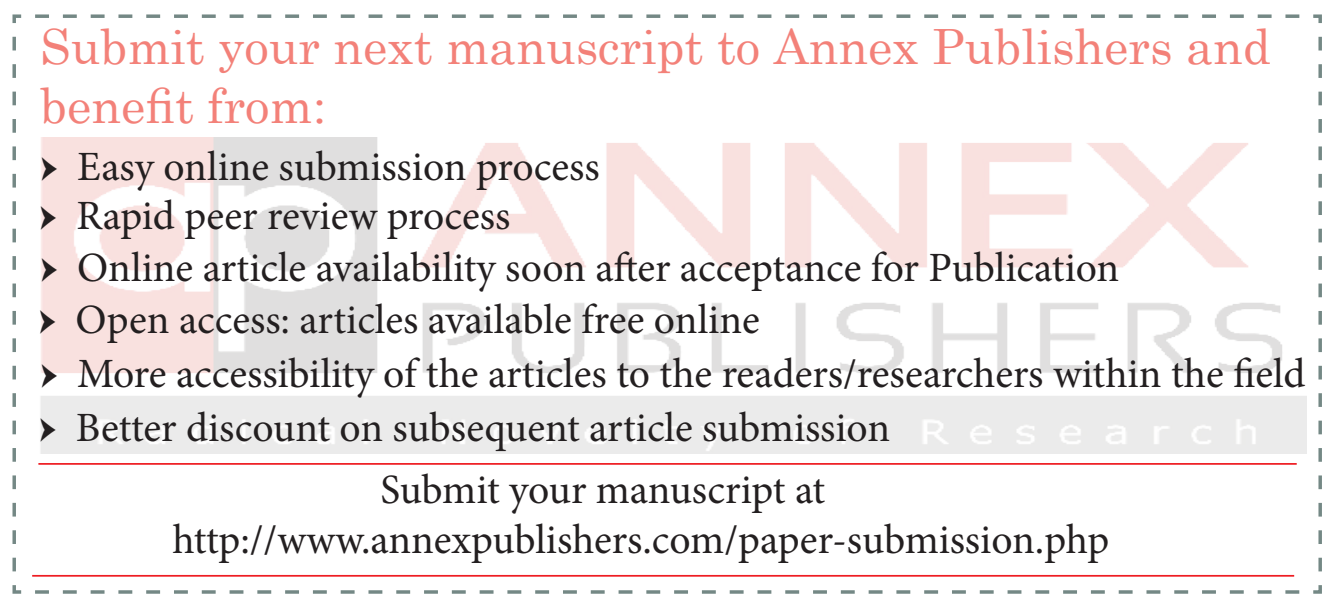

